\title{
Aspergiloma como sequela de tuberculose pulmonar: relato de caso
}

\author{
Aspergiloma as a sequel of pulmonary tuberculosis: case report
}

Aspergiloma como secuela de la tuberculosis pulmonar: reato del caso

Rafael Jose Capuchinho Rocha ${ }^{1 *}$, Julliany Queiroz Borges², Luisa Thainã Pimenta Rocha², Tayná Gonçalves Prado², Camila Teles Gonçalves², João Guilherme Dorneles Ferraz², Fernanda Araújo $\mathrm{Sá}^{3}$, Jordana Figueiredo Amim4, Paolla Dorneles Ferraz Sousa ${ }^{4}$, Evandro Barbosa dos Anjos ${ }^{2}$.

\section{RESUMO}

Objetivo: Relatar um caso de aspergiloma como sequela de tuberculose pulmonar em um paciente jovem e imunocompetente. Relato de Caso: Paciente masculino, 24 anos, trabalhador rural. Admitido em hospital para tratamento cirúrgico do aspergiloma pulmonar, apresentando quadro de tosse seca há quatro meses associada a episódios de hemoptise volumosa. Histórico de tuberculose pulmonar no ano anterior adequadamente tratada. Trazia tomografia de tórax revelando lesão escavada de paredes espessas, no segmento apicoposterior do lobo superior esquerdo, contendo nodulação sólida de contornos bocelados no interior, móvel à mudança de decúbito, sugerindo colonização fúngica sobrejacente. Trazia ainda baciloscopia de escarro e sorologias anti-HIV 1 e 2 negativas. Relatou tratamento com Itraconazol 200mg 12/12 horas por 20 dias há cerca de dois meses, sem melhora clínica. Durante internação foi submetido à lobectomia superior esquerda. Admitido em CTI no pósoperatório imediato, onde permaneceu por três dias. Paciente apresentou boa evolução, seguido por alta hospitalar no nono dia pós-operatório. Considerações Finais: $O$ aspergiloma pulmonar deve ser considerado em todos os pacientes com história de alteração da arquitetura pulmonar, especialmente àquelas produzida por tuberculose, que apresentem uma história respiratória caracterizada por hemoptise. Considera-se o tratamento cirúrgico como primeira escolha incluindo em pacientes assintomáticos.

Palavras-chave: Aspergilose, Tuberculose pulmonar, Hemoptise, Lobectomia.

\begin{abstract}
Objective: To report a case of aspergilloma as a sequel to pulmonary tuberculosis in a young and immunocompetent patient. Case Report: Male, 24-years-old, tiller. Admitted at the hospital for surgical treatment of pulmonary aspergilloma, presenting dry cough for four months associated with episodes of massive hemoptysis. History of pulmonary tuberculosis in previous year adequately treated. He had a chest tomography showing a hollowed-out lesion with thick walls, in the posterior apex segment of the left upper lobe, containing a solid nodulation of contoured contours in the interior, movable in the decubitus position, suggesting overlying fungal colonization. He also had sputum smear microscopy and anti-HIV serologies 1 and 2 negatives. Reported treatment with Itraconazole 200mg 12/12hours during 20 days for about two months, with no clinical improvement. During hospitalization, he underwent a left upper lobectomy. Admitted to the ICU in the immediate postoperative period, where he presented a good evolution, followed by hospital discharge on the ninth postoperative day. Final Considerations: Pulmonary aspergilloma should be considered in all patients with a history of altered pulmonary architecture, especially those produced by tuberculosis, who have a respiratory history characterized by hemoptysis. Surgical treatment is considered the first choice, including in asymptomatic patients.
\end{abstract}

Key words: Aspergillosis, Pulmonary tuberculosis, Hemoptysis, Lobectomy.

\section{RESUMEN}

Objetivo: Reportar un caso de aspergiloma como secuela de tuberculosis pulmonar en paciente joven e inmunocompetente. Reato del caso: Paciente masculino, 24 años, trabajador rural. Ingresado em el hospital para

\footnotetext{
${ }^{1}$ Hospital Felício Rocho (HFR), Belo Horizonte - Minas Gerais. *E-mail: rafacap1994@ hotmail.com

${ }^{2}$ Faculdades Unidas do Norte de Minas (FUNORTE), Montes Claros - Minas Gerais.

${ }^{3}$ Hospital Madre Teresa, Belo Horizonte - Minas Gerais.

${ }^{4}$ Universidade Federal dos Vales Jequitinhonha e Mucuri (UFVJM), Diamantina - Minas Gerais.
} 
tratamento quirúrgico de aspergiloma pulmonar, que presenta tos seca de cuatro meses asociada a episodios de hemoptisismasiva. Historia de tuberculosis pulmonar em el año anterior adecuadamente tratada. Se le realizo tomografía de tórax que mostró una lesión ahuecada de paredes gruesas, en segmento apicoposterior del lóbulo superior izquierdo, conteniendoun nódulo sólido de contornos boceladosenel interior, movibleen decúbito, sugiriendo colonización fúngica suprayacente. También tenía baciloscopia de esputo y serologías anti-VIH 1 y 2 negativas. Refirió tratamento com itraconazol $200 \mathrm{mg} \mathrm{12/12} \mathrm{horas} \mathrm{durante} 20$ díashace dos meses, sin mejoría clínica. Durante la hospitalización, se sometió a lobectomía superior izquierda. Ingresóen UCI em el postoperatorio inmediato, donde permaneció ters días. El paciente presentó buena evolución, seguido de alta hospitalaria al noveno día postoperatorio. Consideraciones finales: Se debe considerar el aspergiloma pulmonar en todos los pacientes con antecedentes de arquitectura pulmonar alterada, especialmente aquellos producidos por tuberculosis, que tienen antecedentes respiratorios caracterizados por hemoptisis. El tratamento quirúrgico se considera la primeraopción, incluso en pacientes asintomáticos.

Palabras clave: Aspergilosis, Tuberculosis pulmonar, Hemoptisis, Lobectomía

\section{INTRODUÇÃO}

O Aspergillus sp. é um fungo de distribuição universal, podendo ser encontrado no ar, solo, materiais em decomposição, paredes úmidas e água. Produzem infecções contagiosas, esporádicas ou oportunistas (TORALE M, et al., 2010). Das mais de 900 espécies existentes, poucas são patógenas para os seres humanos, sendo as mais frequentes: A. fumigatus(65-75\%), A. flavus(05-10\%), A. niger(1,5-03\%), $A$. terrus(02-03\%) (BOSCO MENDOZA C, et al., 2015; MARCHIORI E, et al., 2019).

A real incidência desta patologia não é conhecida, mas sabe-se que houve aumento da taxa nos últimos anos, devido ao incremento da expectativa de vida e à maior sobrevida dos pacientesimunocomprometidos (TORALE M, et al., 2010). É pouco frequente em pacientes imunocompetentes, devendo ser suspeitada diante de um quadro com evolução crônica (FLORES-PONCE L, ARTEAGA-SARMIENTO P, 2018).

Os macrófagos alveolares e os neutrófilos possuem grande capacidade de destruição dos esporos; por isso que a má funcionalidade das mesmas é fator de risco para esta infecção, sendo especialmente significativa quando contagem neutrofílica $<500$ por mm (BRANCO BPC, et al., 2014; TORALE M, et al., 2010;RESTREPO-GUALTEROS SM, et al., 2015).

Pode gerar comprometimento em inúmeros órgãos, sendo o pulmão o principal órgão afetado.A rota usual da infecção é por inalação e o tempo de incubação varia de dias a meses, a depender da espécie (BOSCO MENDOZA C, et al., 2015; FLORES-PONCE L, ARTEAGA-SARMIENTO P, 2018). A infecção pulmonar surge como resultado da colonização pelo fungo em cavidades, cistos ou bolhas pré-existentes, como consequência de diversas enfermidades crônicas, como tuberculose (92\% casos), fibrose cística, bronquiectasias, sarcoidose, neoplasias, espondilite anquilosante e infarto pulmonar. Geralmente não há invasão do parênquima circundante ou de vasos sanguíneos em permeio e o comprometimento predomina nos lobos superiores; bilateral em $05-10 \%$ dos casos (TORALE M, et al., 2010).

A maioria dos casos é assintomática. Quando sintomático, o quadro clínico dependerá da virulência do fungo, da quantidade de exposição e do estado imunológico do paciente, condicionando diferentes formas de apresentação: aspergilosebroncopulmonar alérgica, granulomatose broncocêntrica, aspergilose pulmonar invasiva, traqueobronquite aspergilar, aspergilose pulmonar necrotizante crônica, aspergilomamicematoso (TORALE M, et al., 2010). A queixa mais comum, com incidência de $80 \%$, é a hemoptise, causada pela dilatação local da vasculatura exposta ou destruição dos vasos intracavitários e, quando presente, apresenta $30 \%$ das causas de morte (VALDIVIA VHZ, et al., 2015). Pode haver outros sintomas, como tosse crônica e dispneia (TORALE M, et al., 2010).

O diagnóstico de aspergilose de foco pulmonar requer quadro clínico compatível e antecedentes de sequelas pulmonares, sorologia, cultura de escarro (positivo em $50 \%$ casos), sorologias com IgG (positivo em mais de 90\% casos) e imagens radiológicas sugestivas (sinal de Monod) (VALDIVIA VHZ, et al., 2015; TORALE M, et al., 2010; BRANCO BPC, et al., 2014). A diferenciação das espécies não é mandatória, mas uma característica da infecção por $A$. nigerque é fundamental para o diagnóstico é a presença de cristais de oxalato de cálcio, detectados por exame anatomopatológico (MARCHIORI E, et al., 2019). 
Os principais diagnósticos diferenciais são tuberculose pulmonar e malignidade, que devem ser pensadas em todos os pacientes que apresentem hemoptise (FLORES-PONCE L, ARTEAGA-SARMIENTO P, 2018). Para tal, a radiografia se mostra muito útil: uma mudança de posição do nódulo intracavitário quando o paciente muda de posição é um sinal valioso para o diagnóstico de aspergiloma. Portanto, a avaliação clássica de aspergiloma por tomografia de tórax inclui imagens obtidas em decúbito dorsal e ventral a fim de se demonstrar se a massa central está solta ou presa à parede da cavidade. Diferentemente da bola fúngica, câncer pulmonar cavitário e artrite reumatoide estão fixados à parede da cavidade. $O$ realce da massa pelo meio de contraste nas imagens de tomografia de tórax também pode ajudar nesta diferenciação (MARCHIORI E, et al., 2015).

Há a opção de tratamento clínico (antifúngico) e/ou cirúrgico e a decisão da melhor abordagem é individualizada. Para tomada de decisão, é preciso entender que a penetração do agente anti-fúgico na cavidade é escassa e, por isso, em muitos casos, a única opção viável é a ressecção do parênquima pulmonar colonizado (TORALE M, et al., 2010; KASPRZYK M, et al., 2017).

Grandes benefícios foram observados após tratamento cirúrgico em pacientes com hemoptises recorrentes ou com pelo menos um episódio de hemoptise maciça (TORALE M, et al., 2010; KASPRZYK M, et al., 2017). Em pacientes de alto risco cirúrgico, pode-se realizar embolizações das artérias brônquicas com microesferas, podendo aliviar o quadro de sangramento, embora possua altas taxas de recidiva (TORALE M et al., 2010; SETIANINGRUM F, et al., 2020). Contudo, por se tratarem de hemoptises graves e/ou recorrentes em cerca de $85 \%$ casos, o tratamento clínico fica limitado a pacientes com reserva funcional pulmonar diminuída, sendo o Voriconazol a droga de escolha (CORREIA SS, et al., 2014; VALDIVIA VHZ, et al., 2015 ; RESTREPO-GUALTEROS SM et al., 2015).

Dentre as complicações pós-operatórias devemos destacar a infecção pleural (a mais comum), vazamentos de ar, pneumotórax persistente, TEP e insuficiência respiratória. A qualidade do parênquima pulmonar remanescente é um dos mais importantes fatores prognósticos e suas características no pós operatório depende não só da expertise do cirurgião, mas também do grau de comprometimento (tamanho de área infectada) e região pulmonar acometida (SETIANINGRUM F, et al., 2020; KASPRZYK M, et al., 2017). Por isso, visando à redução do processo infeccioso, em alguns casos, indica-se uso de antifúngicos por três a seis meses antes da cirurgia (SETIANINGRUM F, et al., 2020).

Considera-se o tratamento cirúrgico como primeira escolha nos casos de AP, inclusive em pacientes assintomáticos, aumentando a sobrevida para $84 \%$ ao prevenir a recidiva da hemoptise. O tratamento medicamentoso com antifúngicos está limitado a pacientes com reserva funcional pulmonar diminuída (CORREIA SS, et al, 2014; VALDIVIA VHZ, et al., 2015)

O objetivo deste artigo é relatar um caso de AP como sequela de Tuberculose Pulmonar, em um paciente jovem e imunocompetente, enfatizando a importância do diagnóstico precoce como medida preventiva de complicações, insucesso cirúrgico e óbito.

\section{DETALHAMENTO DO CASO}

O presente artigo teve o projeto avaliado pelo Comitê de Ética e Pesquisa da Funorte, com número do parecer de aprovação 2.403.773.

Paciente do sexo masculino, 24 anos, natural de Montes Claros, trabalhador rural. Admitido em serviço hospitalar para realização de tratamento cirúrgico do AP, apresentando quadro de tosse seca com piora noturna e ao amanhecer, com evolução de quatro meses e episódios de hemoptise volumosa concomitante. Como antecedente de importância, foi diagnosticado com Tuberculose Pulmonar no ano anterior, realizado tratamento com esquema RHZE por 6 meses (Rifampicina, Isoniazida, Pirazinamida e Etambutol por 2 meses, mais 4 meses de Rifampicina e Isoniazida).

Ao exame, apresentava-se em bom estado geral, exame físico e revisão laboratorial do risco cirúrgico sem alterações. Trazia consigo exames de imagem solicitados em consultas prévias. Radiografia de tórax que evidenciou opacidades heterogêneas no terço superior e médio do pulmão esquerdo (Figura 1), medindo 
cerca de $5,0 \mathrm{~cm}$, redução volumétrica pulmonar e leve elevação do hilo pulmonar deste lado. Tomografia de tórax revelando lesão escavada de paredes espessas, localizada no segmento apicoposterior do lobo superior esquerdo (Figura 2), contendo nodulação sólida de contornos bocelados em seu interior, móvel à mudança de decúbito, sugerindo colonização fúngica sobrejacente. Espessamento/retração pleural adjacente; bronquiectasias cilíndricas e varicosas à esquerda; retração hilar e redução do volume pulmonar ipsilateral; linfonodos mediastinais, medindo até $0,9 \mathrm{~cm}$ no seu menor eixo axial, provavelmente de natureza reacional e atelectasia do segmento lingular inferior.

Figura 1 - Radiografia de Tórax em incidências PA e Perfil apresentando opacidades heterogêneas no pulmão esquerdo, com redução volumétrica $\mathrm{e}$ leve elevação do hilo.

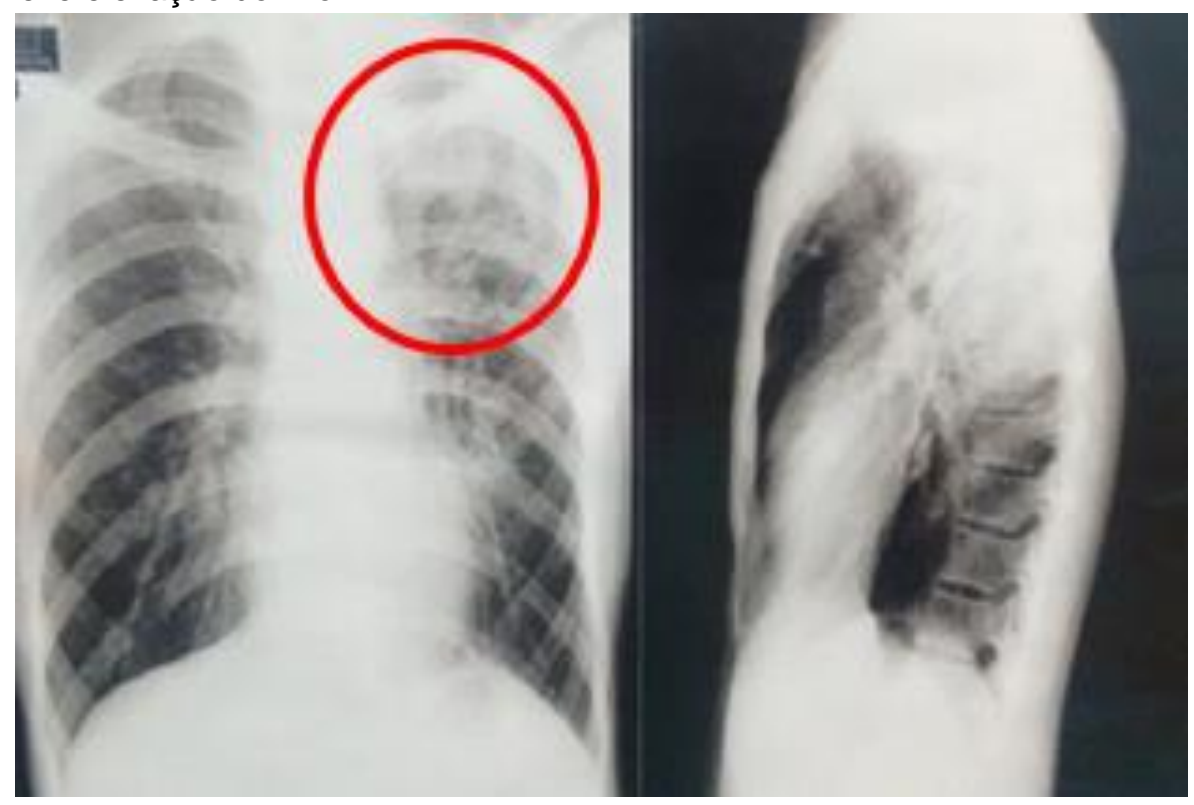

Fonte: Rocha RJC, et al., 2020.

Figura 2 - Tomografia de tórax evidenciando lesão escavada de paredes espessas com nodulação sólida de contornos bocelados. Atelectasia do segmento lingular inferior.

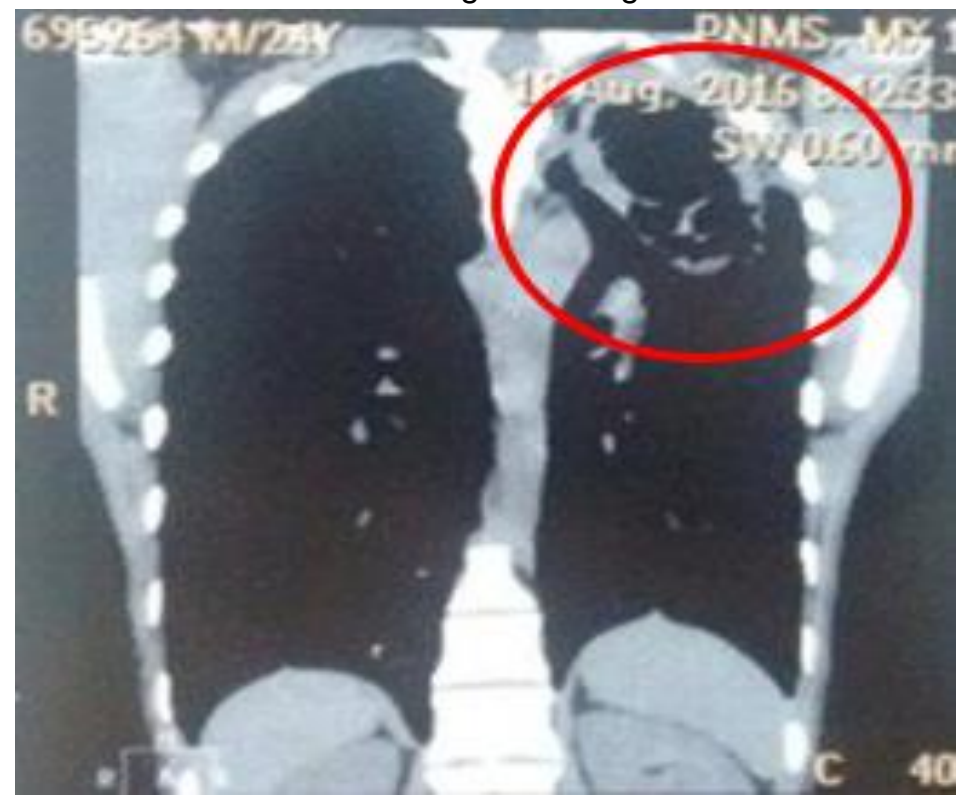

Fonte: Rocha RJC, et al., 2020. 
O paciente trazia ainda uma espirometria que revelava leve distúrbio ventilatório restritivo e baciloscopia de escarro negativa. Também portava sorologia anti-HIV 1 e 2, ambas negativas. O mesmo relatou tratamento medicamentoso com Itraconazol 200mg 12/12h por 20 dias há cerca de dois meses, mantendo os episódios de hemoptise mesmo após tal tratamento. No dia seguinte a internação, foi realizada lobectomia superior esquerda, com colocação de dreno de tórax oㅜ 38. Admitido em CTI no pós-operatório imediato, com alta após três dias para enfermaria cirúrgica. Paciente continuou aos cuidados da cirurgia torácica, com boa evolução em enfermaria foi retirado o dreno de tórax, seguido por alta hospitalar no nono dia pós-operatório.

\section{DISCUSSÃO}

O Aspergiloma é uma formação de bola fúngica pulmonar intracavitária resultante da colonização de um espaço aéreo pré-formado ou natural (SILVA PSMP, et al., 2014). A cura da tuberculose pela quimioterapia tornou possível a sobrevivência de muitos pacientes com cavidades tuberculosas pulmonares saneada e, por isso, atualmente, é a principal condição predisponente para surgimento do Aspergiloma (BRANCO BPC, et al., 2014; SETIANINGRUM F, et al., 2020). No caso descrito, o paciente apresentava em seu histórico, diagnóstico de tuberculose, realizado esquema de tratamento específico, tendo evoluído para a cura e doença inativa. Comprovada pela pesquisa de BAAR em escarro negativa.

A maioria dos indivíduos com Aspergilose Pulmonar é assintomática. Dos pacientes sintomáticos, a queixa mais comum é a hemoptise, presente em cerca de $85 \%$ casos; outras manifestações relevantes são tosse crônica, expectoração, dispneia. baqueteamento digital, astenia e perda de peso (VALDIVIA VHZ, et al., 2015; SILVA PSMP, et al., 2014; TORALE M, et al., 2010). O paciente em questão buscou o serviço de saúde devido a quadro de hemoptise volumosa e tosse não produtiva com piora noturna, quadro compatível com as descrições da literatura. A incoerência clínica baseia-se no fato de que a Aspergilose Pulmonar ocorre, majoritariamente, em pacientes imunocomprometidos (AMORIM DS, et al., 2004). O paciente do relato de caso era jovem, previamente hígido, sem uso de terapias imunossupressoras e com exames laboratoriais sem evidência de imunodepressão, o que contradiz o padrão indicado pela literatura.

$\mathrm{O}$ isolamento do Aspergillus no material da árvore brônquica é somente o primeiro passo numa ampla escala diagnóstica. Há três espécies principais de Aspergillus com clínica significativa: $A$. fumigatus, $A$. niger e A. flavus, sendo a A. fumigatus o agente mais frequente (VALDIVIA VHZ, et al., 2015). No caso acima descrito, não foi possível a identificação do agente etiológico específico, pela indisponibilidade de acesso ao exame, mas é importante salientar que a diferenciação de espécie não é conduta imperativa, pois não repercute em alteração de desfecho clínico e/ou escolha da abordagem terapêutica (BOSCO MENDOZA C, et al., 2015; JUNIOT RLR, et al., 2010).

O diagnóstico se baseia em realização de exame radiográfico do tórax, pela presença de massa ocupando quase toda a cavidade. Geralmente, localiza-se em cavidades presentes nos segmentos apicais e posteriores dos lobos superiores, com formação de um halo aéreo na parte superior (calota aérea, imagem em crescente, "sinal de Monod"). Essas massas intracavitárias geralmente são únicas, com 3 a $5 \mathrm{~cm}$ de diâmetro, hipotransparentes, arredondadas ou ovaladas, e não são fixas, mudando de posição de acordo com o decúbito. A radiografia convencional de tórax em frontal pode não evidenciar a imagem, porém a tomografia computadorizada pode evidenciar a massa dando origem ao aspecto em "laço", e também em "guizo" (BRANCO BPC, et al., 2014). O paciente apresentou radiografia de tórax com opacidades heterogêneas no terço superior e médio do pulmão esquerdo e na tomografia computadorizada observou-se lesão escavada de paredes espessas, contendo nodulação sólida de contornos bocelados em seu interior, móvel à mudança de decúbito, sugerindo colonização fúngica sobrejacente.

O tratamento antifúngico apresenta resultado duvidoso, mesmo em instalação intracavitária, porque esse fármaco não alcança concentrações quimioterápicas adequadas ao nível da lesão. Embora já tenham sido preconizados variados tratamentos clínicos com múltiplos antifúngicos, seus resultados sempre foram precários (BRANCO BPC, et al., 2014). Ao paciente do relato foi prescrito Itraconazol 200mg, no momento do diagnóstico do Aspergiloma Pulmonar, sem melhora do quadro pulmonar até a data da intervenção cirúrgica. O tratamento antifúngico prolongado com Itraconazol ou Voriconazol, talvez até por toda a vida, é 
recomendado para Aspergilose Pulmonar cavitária crônica (CORREIA SS, et al., 2014; VALDIVIA VHZ, et al., 2015; SALES MP, 2009; TORALE M, et al., 2010; KASPRZYK M, et al., 2017).

O tratamento de escolha é a ressecção cirúrgica da área afetada, não havendo, porém, regra definitiva para essa conduta. Os casos são estudados individualmente, avaliando-se a relação risco/benefício (MARCHIORI E, et al., 2019; FLORES-PONCE L, ARTEAGA-SARMIENTO P, 2018). Deve-se considerar idade, estado geral, reserva funcional cardiopulmonar, tipo de cirurgia, volume de hemoptise, condições associadas e tipo de doença predisponente. Os pacientes que não podem se submeter à cirurgia de ressecção recebem medidas paliativas, como cavernostomia, radioterapia e embolização das artérias brônquicas (BRANCO BPC, et al., 2014; TORALE M, et al., 2010; KASPRZYK M, et al., 2017).

O procedimento a ser realizado baseia-se nas características da cavidade e pode ser classificado em dois tipos: AP simples, quando a cavidade é cercada por tecido pulmonar saudável (localizado) e AP complexo, quando a cavidade é cercada por parênquima destruído ou lesões difusas com envolvimento pleural (BOSCO MENDOZA C, et al., 2015). A indicação de pneumectomia é frequente nos casos de AP complexo, uma vez que, geralmente, as lesões pulmonares são extensas (SILVA PSMP, et al., 2014).

No caso apresentado, após avaliação do exame de espirometria que evidenciava leve distúrbio ventilatório restritivo com prova broncodilatadora negativa, o corpo clínico-cirúrgico optou pela realização da lobectomia, embora, exames de imagem mostrassem espessamento/retração pleural adjacente e redução do volume pulmonar ipsilateral. A lobectomia provou ser o procedimento de escolha neste caso, além de ser um procedimento seguro em comparação com outras séries, permitindo recuperação mais rápida, manutenção da superfície de difusão e melhora da sobrevida (VALDIVIA VHZ, et al., 2015; DOS SANTOS VM, 2016).

Tecnicamente as ressecções são difíceis, existe fibrose intensa instalada ao redor da cavidade, obliteração do espaço pleural e ausência de fissuras interlobares, hilo pulmonar enrijecido, intensa circulação colateral e artérias brônquicas aumentadas e tortuosas (SILVA PSMP, et al., 2014; MARCHIORI E, et al., 2015 ; RESTREPO-GUALTEROS SM, et al., 2015 ).

As complicações operatórias mais comuns são: escape de ar prolongado, espaço pleural residual, hemorragia, fístula broncopleural, empiema e morte e estão diretamente relacionadas à dificuldade técnica no momento da ressecção cirúrgica em estádios avançados da doença (VALDIVIA VHZ, et al., 2015; SILVA PSMP, et al., 2014; MARCHIORI E, et al., 2015). O paciente, após a cirurgia, foi admitido em CTI, hemodinamicamente estável, evoluindo sem queixas e com retirada de dreno após redução do escape líquido, melhora clínica e radiológica, permanecendo sem complicações, tendo uma boa resposta possivelmente justificada pela cirurgia precoce.

A partir da análise do relato de caso e da revisão de literatura, conclui-se que o AP deve ser considerado em todos os pacientes com história de alteração da arquitetura pulmonar, especialmente àquelas produzida por tuberculose, que apresentem uma história respiratória caracterizada por hemoptise. Considera-se o tratamento cirúrgico como primeira escolha nos casos de AP, incluindo os pacientes assintomáticos. A dificuldade técnica durante a ressecção cirúrgica nos estágios avançados da enfermidade está associada com um maior número de complicações durante e após a cirurgia, enquanto a cirurgia precoce está relacionada a um menor número de complicações. Por isso, se recomenda melhorar os esforços dirigidos ao diagnóstico e tratamento precoce, apesar das dificuldades pelas múltiplas apresentações clínicas.

\section{REFERÊNCIAS}

1. AMORIM DS, et al. Infecções por Aspergillus spp: aspectos gerais. Pulmão RJ 2004 Abr-Jun; 13(2): 111-18.

2. BOSCO MENDOZA C, et al. Inusual presentación infecciosa oportunista em pulmónsecuelar: reporte de um caso clinico. Rev Am Med Resp 2015 Dez; 4:355-58.

3. BRANCO BPC, et al. Colonização intracavitária pulmonar por aspergillus: análise de 190 casos. J Bras Med 2014 Dez; 102(6).

4. CORREIA SS, et al. Cirurgia no aspergiloma pulmonar: experiência mono-institucional. Acta Med Port 2014 Jul-Ago; 27(4): 417-21. 
5. DOS SANTOS VM. Pulmonaryaspergilloma. Neumol. cir. torax, México, v. 75, n. 2, p. 191, jun. 2016.

6. FLORES-PONCE L, ARTEAGA-SARMIENTO P. Aspegilosis pulmonar crónica cavitada. Med. interna Méx. vol.34 no.1 Ciudad de México ene./feb. 2018.

7. JUNIOT RLR, et al. Tratamento cirúrgico de aspergiloma pulmonar. J braspneumol 2010 Nov-Dez; 36(6): 779-83.

8. KASPRZYK M, et al. Surgicaltreatment for pulmonaryaspergilloma - early and long-termresults. Kardiochirurgia $\mathrm{i}$ Torakochirurgia Polska 2017.

9. MARCHIORI E, et al. Intracavitarynodule in active tuberculosis: differentialdiagnosis of aspergilloma. J. bras. pneumol., São Paulo, v. 41, n. 6, p. 562-563, Dec. 2015.

10. MARCHIORI E, et al. Massa intracavitária calcificada: uma apresentação rara de aspergiloma. J. bras. pneumol., São Paulo, v. 45, n. 2, e20180396, 2019.

11. RESTREPO-GUALTEROS SM, et al. Aspergilosis pulmonar invasiva: reporte de un caso. Biomédica, Bogotá, v. 35, n. 2, p. 171-176, June 2015.

12. SALES MP. Aspergilose: do diagnóstico ao tratamento. J.braspneumol 2009 Dez; 35(12): 1238-244.

13. SETIANINGRUM F, et al. Clinicaloutcomes of patients with chronicpulmonaryaspergillosismanagedsurgically. Eur J CardiothoracSurg 2020.

14. SILVA PSMP, et al. Tratamento do aspergiloma pulmonar complexo por cavernostomia. RevColBrasCir 2014; 41(6): 406-11.

15. TORALE M, et al. Aspergiloma pulmonar bilateral. Arch. Med Int, Montevideo, v. 32, n. 2-3, p. 53-56, jun. 2010.

16. VALDIVIA VHZ, et al. Tratamiento quirúrgico del aspergiloma pulmonar: experiencia de 10 años em el instituto nacional de enfermedades respiratórias. NeumolCirTorax 2015 Out-Nov; 74(4): 240-6. 